\title{
Effect of Continuous Aerobic Exercise on Nerve Growth Factor in
}

\section{Diabetic Rats}

\author{
Mahnaz Omidi i, ${ }^{1}$, Mohsen Ghanbarzadeh ${ }^{1}$, Masoud Nikbakht ${ }^{1}$, Abdolhamid Habibi ${ }^{1}$ and Rouhola \\ Ranjbar $^{1}$ \\ ${ }^{1}$ Department of Exercise Physiology, Faculty of Sport Sciences, Shahid Chamran University of Ahvaz, Ahvaz, Iran \\ "Corresponding author: Department of Exercise Physiology, Faculty of Sport Sciences, Shahid Chamran University of Ahvaz, Ahvaz, Iran. Tel: +98-9183447698, Email: \\ m2omidi@yahoo.com \\ Received 2018 October 20; Revised 2019 January 27; Accepted 2019 February 03.
}

\begin{abstract}
Background: Neurogenesis is the process by which nervous system cells, known as neurons, are produced by neural stem cells. Objectives: This survey aimed to evaluate the effect of long-term aerobic activity on the nerve growth factor (NGF) in diabetic rats. Methods: We divided 84 rats into four groups: (1) healthy control (HC), (2) healthy practice (HP), (3) diabetic control (DC), and (4) diabetic practice (DP). They practiced for 12 weeks in three sessions per week.

Results: Based on the outcome of the survey, there was no difference in the level of NGF after one step of exercise ( $P=0.860)$, but there was a significant difference in other steps of practice $(P=0.001)$. Also, there was a significant difference in all steps $(4$ th, 8 th and 12th weeks of practice $)(P=0.001)$, while the eighth weeks and 12th weeks did not differ significantly $(P=0.198)$.

Conclusions: In the whole steps (all weeks of practice), the level of NGF was lower in the healthy control and diabetic control groups than in the control practice and diabetic practice groups. In other words, the higher incidence of diabetes mellitus, and the more time gone (from 4th to 8th and then 12th week), the higher decrease in the level of NGF occurred, so that continuous aerobic exercise could increases the NGF levels.
\end{abstract}

Keywords: Diabetes Mellitus, Nerve Growth Factor, Hippocampus, Long-Term Aerobic Activity

\section{Background}

Diabetes mellitus is a chronic disease caused by the lack of insulin secretion or decreased insulin sensitivity in body cells (1). Over time, it can cause damage to various organs of the body such as the heart and kidneys (2). Recent research suggests a link between Alzheimer's disease (AD) and diabetes so that increased blood glucose may be the cause of $\mathrm{AD}$ and people with diabetes are more at risk of developing $\mathrm{AD}$ than healthy people (3). Alzheimer's disease is a neurodegenerative disorder characterized by the lack of memory and perception and this type of dementia is involved in the daily function and daily life of the individual (4).

Some areas in the brain such as the hippocampus, amygdala, and prefrontal cortex are the areas of learning (5). The distribution of neurotrophic factors is reported in various regions of the brain, especially the hippocampus, which is responsible for memory and learning and plays a key role in maintaining the health of neuronal cells (6). In pathological conditions, the level of neurotrophic factors changes in the brain and the activation of signaling path- ways decreases. Due to the decrease in the level of the nerve growth factor (NGF) protein and its receptor, retrograde axonal transport is reduced (7). In addition, NGF can cause the maturation of young neural cells and it promotes the release of factors in the brain which destroy free radicals and strengthen brain-derived neurotrophic factor (BDNF) (8).

Research has shown that neurogenesis declines more in people with diabetes than in healthy people. Since the discovery of this relationship is related to recent years, a few studies have been done, especially on the effect of exercise training on reducing the $\mathrm{AD}$ in people with diabetes. However, most research has been done merely on $\mathrm{AD}$ and $\mathrm{a}$ few studies have focused on whether people with diabetes are exposed to reduced neurogenesis and hence, the AD. Considering, there are different opinions about the timing of neurogenesis reduction in people with diabetes and there has been little research on the effects of exercise training on reducing neurogenesis in diabetics, we made an attempt to investigate the NGF level in rats several weeks after injecting diabetes and doing long-term aerobic activ- 
ity to survey when the level of NGF decreases.

\section{Objectives}

Therefore, this study aimed to survey the rate of NGF decrease several weeks after injecting diabetes in rats. Moreover, we examined whether several weeks of longterm aerobic activity increase the rate of NGF in diabetic rats.

\section{Methods}

\subsection{Study Design}

The subjects consisted of 84 rats. The environment temperature was $21 \pm 2^{\circ} \mathrm{C}$, the cycle of light and dark was $12 \mathrm{~h} / 12 \mathrm{~h}$, and humidity was $60 \% \pm 5 \%$.

Diabetes induction in our research, diabetes was induced by a combination of nicotine amide and streptozotocin (STZ) (9). Nicotine amide was injected initially; then, STZ was injected intraperitoneally after 15 min (10). After five days, fast blood glucose was measured and its level of $126-400 \mathrm{mg} / \mathrm{dL}$ indicated diabetes (10).

\subsection{Experimental Design}

The study had four groups of 21 rats. At the end of each step of the training protocol, we eliminated seven rats from each group; thus each of the groups received 21 rats. Subjects practiced for 12 weeks in three sessions per week. The practice load was planned by the principle of over loading. In the first week of training, the speed of practice was $12 \mathrm{~m} / \mathrm{min}$.

After the first week, we increased the training speed by one meter per minute every week. From the second to 10th weeks, the practice time increased by $2.20 \mathrm{~min}$ every day regularly. In the end, the training period went from 20 to $80 \mathrm{~min}$. The duration of training was kept constant for the last two weeks; the duration and intensity of exercise remained constant so that the possible changes were the result of the adjustment to exercise rather than the response to the intensity of training in the last sessions. The intensity of activity was determined by speed (11). The period of adaptation to activity in groups was one week, 5 min per day at a speed of 5 to $7 \mathrm{~m} / \mathrm{min}$. Meanwhile, the heating and cooling at the beginning and the end of the protocol were 3 to $5 \mathrm{~min}$ at a speed of $5-7 \mathrm{~m} / \mathrm{min}$ (Figure 1 ).

Analytical methods the level of NGF was measured in the hippocampal tissue of rats by the ELISA method according to the manufacturer's instructions (Zellbio Germany). The dispersion coefficient and sensitivity of this method were $6.9 \%$ and $2.5 \mathrm{ng} / \mathrm{L}$, respectively. Animals were sacrificed by injecting intraperitoneally with a combination of ketamine (40 - $60 \mathrm{mg} / \mathrm{kg}$ body weight) and xylazine (5 - 15 $\mathrm{mg} / \mathrm{kg}$ body weight).

\subsection{Statistical Analysis}

The data were analyzed using repeated-measures ANOVA and the pairs were compared with the Bonferroni correction.

\section{Results}

There was no difference in baseline weight at all stages $(P=0.651,0.584,0.406)$. Nevertheless, the final weight of rats at the end of each stage was different $(\mathrm{P}=0.001)$ (Table 1). The results of the Mauchly's test were not significant for NGF $(P>0.05)$ (Table 2). The results of repeated measures ANOVA showed significant differences in NGF in different steps (Table 3). The results of the Bonferroni test showed significant differences between all weeks of training, while the eighth and 12th weeks did not differ significantly (Table $4)$.

In all three different time periods, the levels of NGF were lower in healthy control and diabetic control groups than in healthy practice and diabetic practice groups. Over time, the level of NGF decreased and long-term aerobic activity could increase the level of NGF (Figure 2).

\section{Discussion}

The findings did not show significant changes in NGF in diabetic rats during the first four weeks of the study (P $=0.860$ ). As a result, diabetes did not appear to have a significant effect on the growth factor in the hippocampus of diabetic rats in the short-term (four weeks). This finding was contradictory to some studies $(7,12-17)$ and similar to a previous study (18). The ineffectiveness of diabetes in the hippocampus was possibly due to the short duration of research (four weeks).

Other findings indicated a significant change in the level of NGF in eight and 12 weeks $(P=0.001)$ so that the level of NGF in HC and DC groups groups reduced eight and 12 weeks after diabetes. This variable showed a significant increase after eight and 12 weeks of aerobic training. This result showed the effect of exercise training on increasing the NGF in diabetic subjects. These findings are similar to previous findings $(7,8,13,16,19)$ and contradictory to some studies $(5,14,15,17)$.

The findings indicated a significant change in the NGF after all steps of training so that four weeks of aerobic training had a significant difference with eight and 12 weeks of training $(\mathrm{P}=0.001)$. This is while there was no significant difference between eight and 12 weeks of training $(P=0.198)$. These results are similar to some studies $(7,8,13$, $16,19)$.

The NGF signaling is independent of microtubules by activating two different secondary messaging systems (20, 


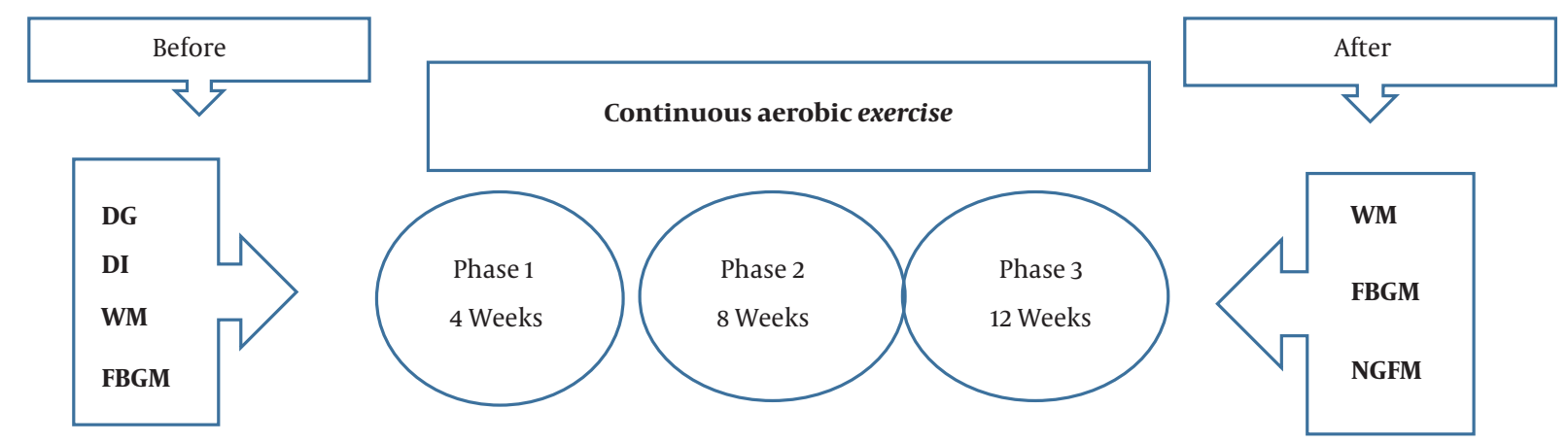

Figure 1. Experimental design. DG, Distribution of groups; DI, diabetes injection; WM, weight mensuration; FBGM, fasting blood glucose mensuration; NGFM, nerve growth factor measurement; Before, before practice; After, after each step.

\begin{tabular}{|c|c|c|c|c|c|}
\hline & HCG & HPG & DCG & DPG & PValue \\
\hline \multicolumn{6}{|l|}{ First step } \\
\hline \multicolumn{6}{|l|}{ BW } \\
\hline Baseline & $277.2 \pm 35.9$ & $271.5 \pm 21.9$ & $282.6 \pm 22.8$ & $267 \pm 24.4$ & 0.751 \\
\hline After step 1 & $281.4 \pm 33.4$ & $272.9 \pm 33.4$ & $215.90 \pm 20.5$ & $251 \pm 30.2$ & 0.002 \\
\hline \multicolumn{6}{|l|}{ FG } \\
\hline Baseline & $87.1 \pm 9.8$ & $92.5 \pm 3.7$ & $367.7 \pm 18.9$ & $369.3 \pm 43.5$ & 0.002 \\
\hline Behind step 1 & $86.3 \pm 9.5$ & $84.7 \pm 5.9$ & $350.2 \pm 100.8$ & $203.5 \pm 111.8$ & 0.002 \\
\hline \multicolumn{6}{|l|}{ Second step } \\
\hline \multicolumn{6}{|l|}{ BW } \\
\hline Baseline & $276.6 \pm 27.1$ & $271.7 \pm 15.3$ & $287 \pm 16.8$ & $265.4 \pm 18.1$ & 0.684 \\
\hline Behind step 2 & $293.4 \pm 21.1$ & $288.7 \pm 16.2$ & $214.2 \pm 44.7$ & $252.4 \pm 28.4$ & 0.002 \\
\hline \multicolumn{6}{|l|}{ FG } \\
\hline Baseline & $89.6 \pm 4.6$ & $88.7 \pm 3.4$ & $353.2 \pm 53.5$ & $352 \pm 52.89$ & 0.003 \\
\hline Behind step 2 & $91.5 \pm 5$ & $72.2 \pm 11.3$ & $419.6 \pm 97.5$ & $213.4 \pm 97.7$ & 0.003 \\
\hline \multicolumn{6}{|l|}{ Third step } \\
\hline \multicolumn{6}{|l|}{ BW } \\
\hline Baseline & $301 \pm 13.8$ & $282.2 \pm 11$ & $287.4 \pm 21.1$ & $279.7 \pm 41$ & 0.306 \\
\hline Behind step 3 & $302.8 \pm 15.9$ & $315.8 \pm 15.4$ & $213.2 \pm 44.3$ & $293.5 \pm 44.4$ & 0.003 \\
\hline \multicolumn{6}{|l|}{ FG } \\
\hline Baseline & $93.1 \pm 9.9$ & $92.2 \pm 6.7$ & $332.5 \pm 61$ & $331.2 \pm 56.2$ & 0.002 \\
\hline Behind step 3 & $92.9 \pm 8.5$ & $78.8 \pm 9.7$ & $455.1 \pm 115.8$ & $91.4 \pm 16.1$ & 0.003 \\
\hline
\end{tabular}

Abbreviations: BW, body weight; DCG, diabetic control group; DPG, diabetic practice group; FG, fasting glucose; HCG, healthy control group; HPG, healthy practice group.

${ }^{a}$ Values are expressed as mean $\pm S D$.

21): one of the involved messaging systems is the MAPK cascade, which functions through Erk phosphorylation, while the other messaging system involves NGF stimulation through activation of the PI3-K/Akt signaling pathway.

Williams et al. (22) observed some ERK and AKT in the hippocampus of aging, NGF-injected rats; here, the phos- phorylated ERK level much increased but the level of Akt remained intact. Researchers have proven that these two various pathways overlap. Thus, it seems to improve cognitive performance by the retrograde transport is accelerated with PI3-K/Akt signaling pathway or MAPK cascade (22). 


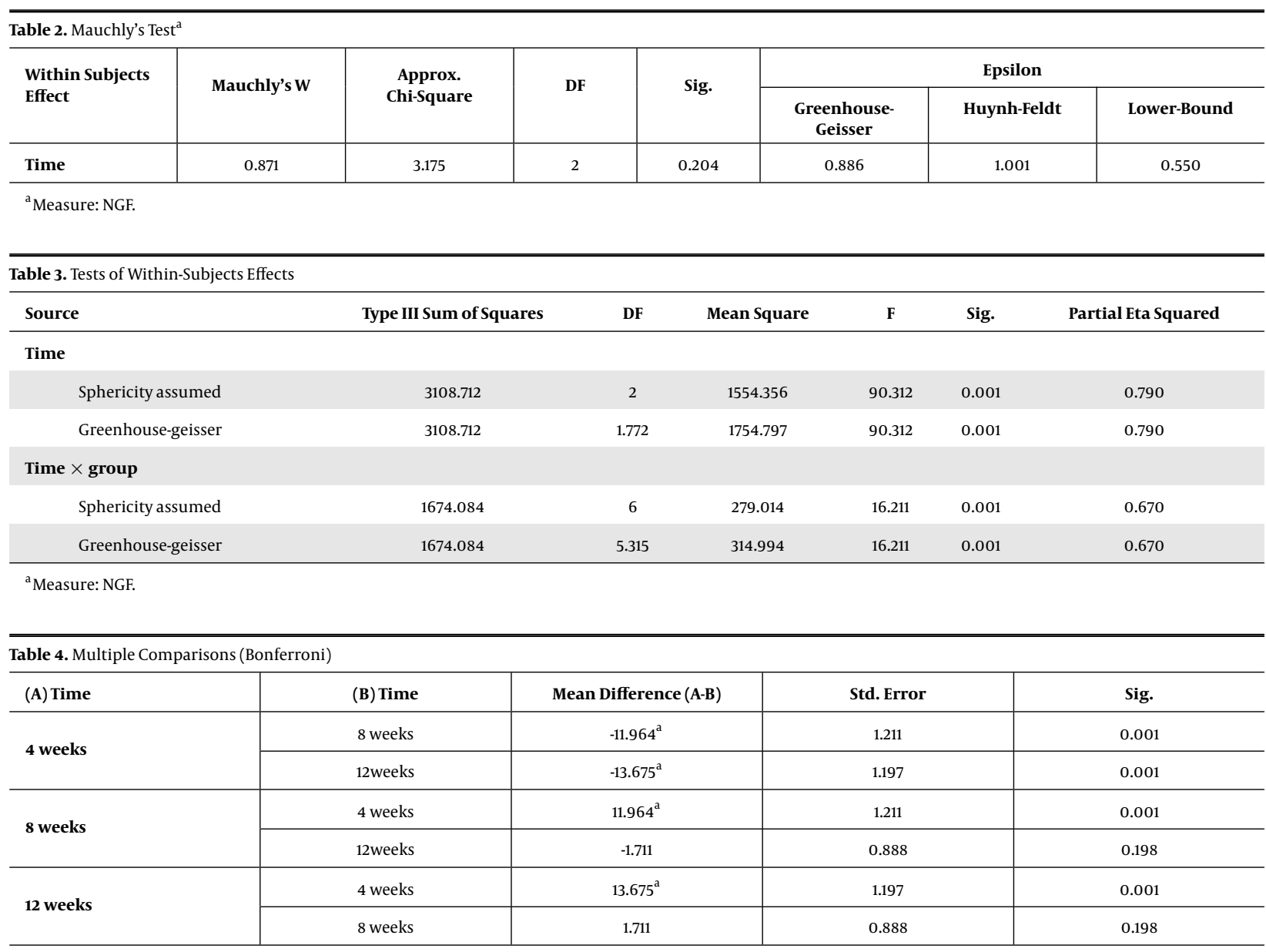

${ }^{\mathrm{a}}$ The mean difference is significant at the 0.05 level.

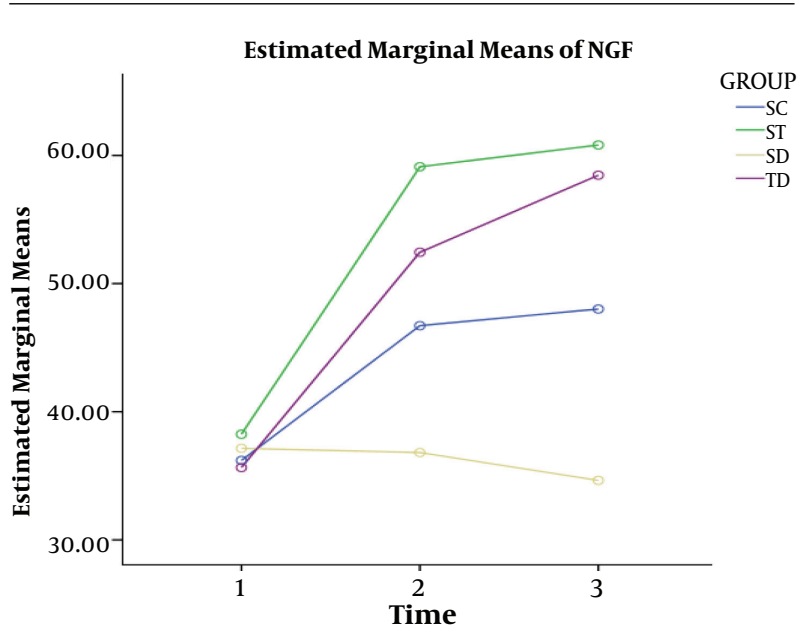

Figure 2. The levels of nerve growth factor in all stages
The PI3-K/Akt pathway interferes with diabetes mellitus. reduction in the retrograde transport of neurotrophins and regular exercise does not disrupt the PI3$\mathrm{K}$ activity, but it can activate MAPK (23). Shen et al. (24) showed that rats with regular physical activity had CREB and phosphorylated MAPK/Erk in their hippocampus. In this study, the levels of p-Erk1/2 were higher in the diabetic exercise group than in the diabetic control group. Besides, the CREB transcription factor was phosphorylated (24). The MAPK/Erk is a transcription factor affecting neuronal plasticity and learning in the hippocampus and it is effective in long-term potentiation (LTP) (25-27).

The NGF activation by ERK during LTP can affect cholinergic neurons, cholinergic neurotransmitters, the hippocampus, and the cerebral cortex (28-30). However, the function of cholinergic neurons is impaired in the elderly and patients with pathologic diseases, and ERK function is eliminated (31). This condition reduces the retrograde transport of NGF because the endocytosis of NGF-tyrosine kinases A (Trk A) is dependent on the ERK activity (32). 
In general, the activation of the PI3K/AKT signaling pathway, the MAPK cascade by Erk decreased and reduces the retrograde transport of neurotrophins, which reduces NGF as a sign of $A D$, over time in the brain of diabetic subjects, and Exercise training induce phosphorylation of CREB as well as phosphorylation of MAPK/Erk in the hippocampus of rats (12).

A limitation of the study was that the basic physiological capacity of rats (heart rate, lactic acid level, $\mathrm{VO}_{2 \max }$, etc.) was not measured.

It is suggested that catecholamines and Insulin-like growth factor 1 (IGF-1) be measured to determine their relationships with exercise training.

\subsection{Conclusions}

We concluded that the more time you have diabetes, the lower your brain NGF levels will be. Therefore, the longterm aerobic activity could increase the level of this variable.

\section{Acknowledgments}

I would like to thanks for their contribution to the development of this research from the members of Exercise Physiology Department, Sport Sciences Faculty, Shahid Chamran University of Ahvaz, Ahvaz, Iran.

\section{Footnotes}

Authors' Contribution: All authors of the article have participated equally.

Conflict of Interests: The authors declare no conflicts of interest regarding the publication of this manuscript.

Ethical Approval: This research was approved by the Ethics Committee of the Shahid Chamran University of Ahvaz and its ethics code was EE/97.24.3.17656/sc.uac.ir.

Funding/Support: This article was supported by Shahid Chamran University of Ahvaz, Ahvaz, Iran.

\section{References}

1. Salehi OR, Hoseini A. The effects of endurance trainings on serum BDNF and insulin levels in streptozotocin-induced diabetic rats. Neurosci J Shefaye Khatam. 2017;5(2):52-61. doi: 10.18869/acadpub.shefa.5.2.52

2. Handsaker JC, Brown SJ, Bowling FL, Maganaris CN, Boulton AJ, Reeves ND. Resistance exercise training increases lower limb speed of strength generation during stair ascent and descent in people with diabetic peripheral neuropathy. Diabet Med. 2016;33(1):97-104. doi: 10.1111/dme.12841. [PubMed: 26108438].

3. Macauley SL, Stanley M, Caesar EE, Yamada SA, Raichle ME, Perez R, et al. Hyperglycemia modulates extracellular amyloid-beta concentrations and neuronal activity in vivo. J Clin Invest. 2015;125(6):24637. doi: 10.1172/JCI79742. [PubMed: 25938784]. [PubMed Central: PMC4497756].
4. Spranger M, Lindholm D, Bandtlow C, Heumann R, Gnahn H, NaherNoe M, et al. Regulation of nerve growth factor (NGF) Synthesis in the rat central nervous system: Comparison between the effects of interleukin-1 and various growth factors in astrocyte cultures and in vivo. Eur J Neurosci. 1990;2(1):69-76. doi: 10.1111/j.14609568.1990.tb00382.x. [PubMed: 12106104].

5. Chen HI, Lin LC, Yu L, Liu YF, Kuo YM, Huang AM, et al. Treadmill exercise enhances passive avoidance learning in rats: The role of downregulated serotonin system in the limbic system. Neurobiol Learn Mem. 2008;89(4):489-96. doi: 10.1016/j.nlm.2007.08.004. [PubMed: 17892954].

6. Ossali A, Eskandari M. Effect of three-month aerobic training on serum levels of BDNF and TNF- $\alpha$ in women with metabolic syndrome on 24 women with metabolic syndrome. Med J Mashhad univ Med Sci. 2016;59(4):242-51.

7. Chae $\mathrm{CH}$, Jung SL, An SH, Park BY, Wang SW, Cho IH, et al. Treadmill exercise improves cognitive function and facilitates nerve growth factor signaling by activating mitogen-activated protein kinase/extracellular signal-regulated kinase1/2 in the streptozotocininduced diabetic rat hippocampus. Neuroscience. 2009;164(4):166573. doi: 10.1016/j.neuroscience.2009.09.075. [PubMed: 19800940].

8. Chae CH, Jung SL, An SH, Jung CK, Nam SN, Kim HT. Treadmill exercise suppresses muscle cell apoptosis by increasing nerve growth factor levels and stimulating p-phosphatidylinositol 3-kinase activation in the soleus of diabetic rats. J Physiol Biochem. 2011;67(2):235-41. doi: 10.1007/s13105-010-0068-9. [PubMed: 21207218].

9. Pierre W, Gildas AJ, Ulrich MC, Modeste WN, Benoit NT, Albert K. Hypoglycemic and hypolipidemic effects of Bersama engleriana leaves in nicotinamide/streptozotocin-induced type 2 diabetic rats. BMC Complement Altern Med.2012;12:264. doi: 10.1186/1472-6882-12-264. [PubMed: 23267560]. [PubMed Central: PMC3546073].

10. Skovso S. Modeling type 2 diabetes in rats using high fat diet and streptozotocin. J Diabetes Investig. 2014;5(4):349-58. doi: 10.1111/jdi.12235. [PubMed: 25411593]. [PubMed Central: PMC4210077].

11. Husamettin V, Evrim C. The effects of 8-week aerobic exercises on the blood lipid and body composition of the owerweight and obese. $\mathrm{Fe}$ males Sci Mov Health. 2010;2:814-20.

12. Cheng H, Hayes J, Hong Y, Feldman E. Uprequlation of nerve growth factor signaling in painful neuropathyof type 2 diabetes. $J$ Pain. 2012;140:35-47.

13. Eslami R, Gharakhanlou R, Kazemi A, Dakhili AB, Sorkhkamanzadeh $\mathrm{G}$, Sheikhy A. Does endurance training compensate for neurotrophin deficiency following diabetic neuropathy? Iran Red Crescent Med J. 2016;18(10). e37757. doi: 10.5812/ircmj.37757. [PubMed: 28184326]. [PubMed Central: PMC5291940].

14. Moser KV, Reindl M, Blasig I, Humpel C. Brain capillary endothelial cells proliferate in response to NGF, express NGF receptors and secrete NGF after inflammation. Brain Res. 2004;1017(1-2):53-60. doi: 10.1016/j.brainres.2004.05.013. [PubMed:15261099].

15. Pittenger G, VinikA. Nerve growth factor and diabetic neuropathy. Exp Diabesity Res. 2003;4(4):271-85. doi: 10.1155/EDR.2003.271. [PubMed: 14668049]. [PubMed Central: PMC2478610].

16. Roh HT, Cho SY, Yoon HG, So WY. Effect of exercise intensity on neurotrophic factors and blood-brain barrier permeability induced by oxidative-nitrosative stress in male college students. Int J Sport Nutr Exerc Metab. 2017;27(3):239-46. doi: 10.1123/ijsnem.2016-0009. [PubMed: 27997261].

17. Ziegler D, Siekierka-Kleiser E, Meyer B, Schweers M. Validation of a novel screening device (NeuroQuick) for quantitative assessment of small nerve fiber dysfunction as an early feature of diabetic polyneuropathy. Diabetes Care. 2005;28(5):1169-74. doi: 10.2337/diacare.28.5.1169. [PubMed: 15855584].

18. Cunha JM, Funez MI, Cunha FQ, Parada CA, Ferreira SH. Streptozotocin-induced mechanical hypernociception is not dependent on hyperglycemia. Braz J Med Biol Res. 2009;42(2):197-206. doi: 10.1590/s0100-879x2009000200008. [PubMed: 19274348]. 
19. Nowroozi Kakheki R, Saheekh al-Salami A; Sa'abb-o-M. Effect of aerobic exercise and consumption of omega-3 on the level of neuropathy of the hippocampus in healthy male mice with homocysteineinduced Alzheimer's disease. IUMS. 2016;379:392-400.

20. Barker PA, Hussain NK, McPherson PS. Retrograde signaling by the neurotrophins follows a well-worn trk. Trends Neurosci. 2002;25(8):379-81. doi: 10.1016/s0166-2236(02)02199-9. [PubMed: 12127743].

21. Gao Y, Nikulina E, Mellado W, Filbin MT. Neurotrophins elevate cAMP to reach a threshold required to overcome inhibition by MAG through extracellular signal-regulated kinase-dependent inhibition of phosphodiesterase. J Neurosci.2003;23(37):11770-7. [PubMed: 14684879]. [PubMed Central: PMC6740960].

22. Williams B, Granholm AC, Sambamurti K. Age-dependent loss of NGF signaling in the rat basal forebrain is due to disrupted MAPK activation. Neurosci Lett. 2007;413(2):110-4. doi:10.1016/j.neulet.2006.11.040. [PubMed: 17182181]. [PubMed Central: PMC1839982].

23. Cai F, Helke CJ. Abnormal PI3 kinase/Akt signal pathway in vagal afferent neurons and vagus nerve of streptozotocin-diabetic rats. Brain Res Mol Brain Res. 2003;110(2):234-44. doi:10.1016/s0169-328x(02)00652-6. [PubMed: 12591159].

24. Shen H, Tong L, Balazs R, Cotman CW. Physical activity elicits sustained activation of the cyclic AMP response element-binding protein and mitogen-activated protein kinase in the rat hippocampus. Neuroscience. 2001;107(2):219-29. doi: 10.1016/s0306-4522(01)00315-3. [PubMed: 11731096].

25. Atkins CM, Selcher JC, Petraitis JJ, Trzaskos JM, Sweatt JD. The MAPK cascade is required for mammalian associative learning. Nat Neurosci.
1998;1(7):602-9. doi: 10.1038/2836. [PubMed: 10196568].

26. Coogan AN, O'Leary DM, O'Connor JJ. P42/44 MAP kinase inhibitor PD98059 attenuates multiple forms of synaptic plasticity in rat dentate gyrus in vitro. J Neurophysiol. 1999;81(1):103-10. doi: 10.1152/jn.1999.81.1.103. [PubMed: 9914271].

27. English JD, Sweatt JD. Activation of p42 mitogen-activated protein kinase in hippocampal long term potentiation. $J$ Biol Chem. 1996;271(40):24329-32. doi: 10.1074/jbc.271.40.24329. [PubMed: 8798683].

28. Huerta PT, Lisman JE. Bidirectional synaptic plasticity induced by a single burst during cholinergic theta oscillation in CA1 in vitro. $\mathrm{Neu}$ ron. 1995;15(5):1053-63. doi: 10.1016/0896-6273(95)90094-2. [PubMed: 7576649].

29. Jerusalinsky D, Kornisiuk E, Izquierdo I. Cholinergic neurotransmission and synaptic plasticity concerning memory processing. Neurochem Res. 1997;22(4):507-15. doi: 10.1023/a:1027376230898. [PubMed: 9130263].

30. Natsume K, Kometani K. Theta-activity-dependent and -independent muscarinic facilitation of long-term potentiation in guinea pig hippocampal slices. Neurosci Res. 1997;27(4):335-41. doi: 10.1016/s01680102(97)01167-x. [PubMed: 9152046].

31. Miller FD, Kaplan DR. On Trk for retrograde signaling. Neuron. 2001;32(5):767-70. doi: 10.1016/s0896-6273(01)00529-3. [PubMed: 11738023].

32. Howe CL, Valletta JS, Rusnak AS, Mobley WC. NGF signaling from clathrin-coated vesicles: evidence that signaling endosomes serve as a platform for the Ras-MAPK pathway. Neuron. 2001;32(5):801-14. doi: 10.1016/s0896-6273(01)00526-8. [PubMed: 11738027]. 